\title{
Orthogonal control of gene expression in plants using synthetic promoters and CRISPR-based transcription factors
}

Shaunak Kar1,2,\#, Yogendra Bordiya1,4,\#, Nestor Rodriguez¹, Junghyun Kim¹, Elizabeth C. Gardner ${ }^{1,2}$, Jimmy Gollihar ${ }^{3}$, Sibum Sung ${ }^{1, *}$ and Andrew D. Ellington ${ }^{1,2, *}$

${ }^{1}$ Department of Molecular Biosciences, University of Texas at Austin, Austin TX, USA

${ }^{2}$ Center for Systems and Synthetic Biology, University of Texas at Austin, Austin, TX, USA

${ }^{3}$ US Army Research Laboratories-South, Austin, Texas, USA

124 Present address: Life Sciences Solutions group, Thermo Fisher Scientific, Austin, TX, 13 USA

\# Authors contributed equally

* Corresponding authors

Sibum Sung: sbsung@austin.utexas.edu, Andrew D. Ellington: ellingtonlab@gmail.com

\section{Abstract}

Background: The construction and application of synthetic genetic circuits is frequently improved if gene expression can be orthogonally controlled, relative to the host. In plants, orthogonality can be achieved via the use of CRISPR-based transcription factors that are programmed to act on natural or synthetic promoters. The construction of complex gene

27 circuits can require multiple, orthogonal regulatory interactions, and this in turn requires

28 that the full programmability of CRISPR elements be adapted to non-natural and non-

29 standard promoters that have few constraints on their design. Therefore, we have developed synthetic promoter elements in which regions upstream of the minimal $35 \mathrm{~S}$

31 CaMV promoter are designed from scratch to interact via programmed gRNAs with dCas9

32 fusions that allow activation of gene expression. 
34 Results: A panel of three, mutually orthogonal promoters that can be acted on by artificial

35 gRNAs bound by CRISPR regulators were designed. Guide RNA expression targeting

36 these promoters was in turn controlled by either Pol III (U6) or ethylene-inducible Pol II

37 promoters, implementing for the first time a fully artificial Orthogonal Control System

38 (OCS). Following demonstration of the complete orthogonality of the designs, the OCS

39 was tied to cellular metabolism by putting gRNA expression under the control of an

40 endogenous plant signaling molecule, ethylene. The ability to form complex circuitry was

41 demonstrated via the ethylene-driven, ratiometric expression of fluorescent proteins in

42 single plants.

44 Conclusions: The design of synthetic promoters is highly generalizable to large tracts

45 of sequence space, allowing Orthogonal Control Systems of increasing complexity to

46 potentially be generated at will. The ability to tie in several different basal features of

47 plant molecular biology (Pol II and Pol III promoters, ethylene regulation) to the OCS

48 demonstrates multiple opportunities for engineering at the system level. Moreover, given

49 the fungibility of the core $35 \mathrm{~S}$ CaMV promoter elements, the derived synthetic promoters

50 can potentially be utilized across a variety of plant species.

\section{Keywords}

54 Synthetic transcription factor, orthogonal promoter, modular cloning, plant synthetic

55 biology 


\section{Introduction}

59 The field of synthetic biology aims to revolutionize biotechnology by rationally engineering

60 living organisms (1-6). One aspect of rational engineering is to embed biological

61 organisms with complex information processing systems that can be used to control

62 phenotypes $(2,3,7,8)$, often via synthetic gene circuits that can predictability regulate

63 and tune expression of endogenous as well as transgenes (4, 9-11).

64 However the performance of such synthetic genetic circuits is often plagued by unwanted

65 interactions between the circuit components and the host regulatory system, which can

66 lead to loss of circuit function (10). These unprogrammed interactions can be mitigated

67 via the design and use of genetic parts that have minimal cross-talk with the host, creating

68 orthogonal regulatory or orthogonal control systems (OCS) that can further serve as the

69 basis for constructing complex genetic programs with predictable behaviors. In the last

70 two decades an increasing number of well-characterized genetic parts have been

71 combined in circuits capable of complex dynamic behaviors, including bi-stable switches,

72 oscillators, pulse generators, Boolean-complete logic gates $(7,12-15)$. While OCS and

73 the circuits that comprise them were initially characterized in microbial hosts, more

74 recently a significant fraction of them have been constructed and characterized in

75 eukaryotic hosts such as yeast and mammalian cells (12, 16-19). More recently, synthetic

76 transcriptional control elements have begun to be characterized in plants (20-22).

77 While a variety of artificial plant transcription factors containing diverse DNA binding

78 domains and plant-specific regulatory sequences are known $(20,22)$, orthogonal control

79 requires more programmable DNA binding domains and modular regulatory domains (20, 
22-24). To this end, we describe an alternate strategy for the construction of orthogonal

81 transcriptional regulatory elements in plants, powered by a single universal transcriptional

82 factor - dCas9:VP64 which has been shown to work in a wide variety of eukaryotic

83 species, including plants $(16,25,26)$. While this transcription factor has primarily been

84 used for the regulation of endogenous genes (25-27), here we describe a generalizable

85 strategy for the universal design and use of synthetic promoters that rely only on the

86 production of specific gRNAs to program dCas9:VP64, and the use of this set of mutually

87 orthogonal promoters for the bottom-up construction of circuits that show multiplexed

88 control of gene expression.

\section{Design of a modular cloning framework for facile construct assembly}

92 Traditionally the process of construction of these synthetic gene expression systems has

93 relied on time-consuming practices of recombinant DNA technology like design of custom

94 primers, PCR amplification, gel extraction of PCR products. Over the last decade the

95 advent of high-throughput cloning techniques, such as Golden-gate cloning with Type IIS

96 restriction enzymes, has greatly accelerated the design-build-test cycle for the

97 construction and prototyping of synthetic circuits $(7,9,28,29)$. Because the overlaps for

98 assemblies can be modularly specified, multiple parts can be assembled sequentially in

99 a single tube reaction.

100 While a Golden-Gate framework was previously described for the construction of plant 101 expression vectors (30), here we used the highly optimized modular cloning (MoClo)

102 framework, instantiated as a yeast toolkit (YTK) as the basis of our architecture (28). 
103 Recently, beyond yeast expression vectors, this framework has been adapted for the

104 construction of a mammalian toolkit (MTK) (9). Along with both YTK and MTK, a plant

105 toolkit based on the YTK architecture will prove essential for seamlessly porting parts and

106 circuits across diverse eukaryotes. Briefly, in this framework the entire vector is divided

107 into particular 'part' types flanked by Bsal restriction sites followed by a unique ligation

108 site. Promoters, genes and terminators are generally categorized into Type 2, 3 and 4

109 parts respectively where each part type has a unique overhang that dictates the

110 compatibility between part types $(9,28)(\mathbf{F i g} \mathbf{1 A}, \mathbf{S 1 A})$. This preserves the architecture of

111 each transcriptional unit (promoter-gene-terminator). For the assembly of multiple

112 transcriptional units (TU), each transcriptional unit is first cloned into an 'intermediate'

113 vector flanked by connector sequences that dictate the order of the TUs to be stitched

114 together. By using appropriate connectors, each TU can be further assembled into a final

115 expression vector in a single pot reaction (Fig S1B) [20]. This modular approach enables

116 rapid assembly of increasingly complex genetic circuits comprised of multiple

117 transcriptional units.

118 Since Agrobacterium-based transformation has been the staple for plant genetic

119 engineering for decades (31), we used compatible vectors as the basis for our framework,

120 and designed and constructed three YTK-compatible shuttle vectors. Each expression

121 vector contains the pVS1 replicon (an Agrobacterium origin of replication - OriV and two

122 supporting proteins - RepA and StaA) and pBR22 origin for propagation in Agrobacterium

123 and E.coli respectively, and a common antibiotic selection cassette (KanR) that has been

124 shown to be functional in both species (Fig 1B, Materials and Methods) (29, 30). The

125 three constructs otherwise differed in the plant selection marker - BASTA, hygromycin, 
126 and kanamycin. The resistance markers were expressed from the Nos promoter and also

127 contained a Nos terminator (30) (Fig 1B). The backbone also contains a GFP drop-out

128 cassette that allows easy identification of correct assemblies, which should appear as

129 colonies that lack fluorescence $(9,28)$ (Fig 1B).

131 Fluorescence and luminescence reporters are frequently used to study protein 132 localization and interaction in plants and animals (32). To provide these useful reporter 133 parts in the context of our system, we cloned the strong promoter from Cauliflower mosaic 134 virus (35S) as a Type 2 part and its corresponding terminator as a Type 4 part $(33,34)$. 135 These parts can be matched with a number of fluorescent reporter genes (GFP, BFP, 136 YFP and RFP) all as Type 3 parts for robust reporter expression. Combinations of these 137 proteins can also potentially be used for BIFC (Bimolecular Fluorescence 138 Complementation) (35). Similarly, luciferase is commonly used in plant molecular biology 139 to study circadian rhythm (36), test the spatiotemporal activities of regulatory elements 140 (37), and to study the plant immune system $(38,39)$. Therefore we adapted a luciferase 141 gene from Photinus pyralis, commonly known as firefly luciferase (F-luc) (21).

143 Single TUs comprised of a 35S promoter, fluorescent reporter genes and the luciferase 144 gene, and a terminator that serves as a polyadenylation signal were assembled into the 145 Agrobacterium shuttle expression vector (Fig 2A-C). The activity of constructs was 146 assayed using transient expression in Nicotiana benthamiana (30). As expected, we see 147 strong activity of the promoter with the expression of the respective reporter genes (Fig 148 2A-C). In order to diversify the promoters used in circuits (and thereby avoid 
149 recombination and potentially silencing), we also included a well-characterized promoter

150 from the Ti plasmid that drives mannopine synthase (Pmas) (40-43). When the 35S

151 promoter was swapped with Pmas, similar expression levels of YFP were achieved (Fig 152 2D).

153

154 Development of an Orthogonal Control System (OCS) to regulate transgene expression

156 One of the primary difficulties with using synthetic biology principles and methods to

157 engineer organisms, especially in eukaryotes, is that the functionality of synthetic circuits

158 is often plagued by unwanted interactions of the circuit 'parts' with the underlying 159 regulatory machinery of the host (44). As a particularly relevant example, systems 160 developed in the past for transgene expression caused severe growth and developmental 161 defects in Arabidopsis and Nicotiana benthamiana $(45,46)$. Therefore, it is paramount to 162 develop regulatory tools to control transgene expression that minimizes the impact on 163 endogenous plant machinery/physiology, while maintaining the modularity and scalability 164 of synthetic approaches in general.

166 A potential solution to this problem is to develop orthogonal 'parts' that of necessity 167 function independently of endogenous regulation by the host. To this end, we set out to 168 develop a fully integrated Orthogonal Control System (OCS) based on orthogonal 169 synthetic promoters driven by an Artificial Transcription Factor (ATF). We started with 170 the deactivated form of the Cas9 protein (dCas9) fused to the transcriptional activator 171 domain VP64 as a highly programmable ATF $(26,27)$. While dCas9:VP64 has previously 
172 been shown to upregulate the expression of endogenous genes via specific guide RNAs

173 (gRNAs) that target the promoter region upstream of those genes $(25,47)$, this strategy

174 has not been utilized for the construction of a fully orthogonal system in which custom

175 promoters can be similarly regulated. Here we develop a suite of synthetic promoters

176 (pATFs, promoter for Artificial Transcription Factor) in which each promoter has a similar

177 modular architecture: varying number of repeats of gRNA binding sites followed by a

178 minimal 35 S promoter $(33,34)$. This system is inherently scalable, since new binding

179 sites bound by new gRNAs can be built at will. The complete list of parts (promoters,

180 genes and terminators) is provided in Supplementary Table 1.

182 We initially varied the number of gRNA binding sites (3 and 4) upstream of the minimal

183 35S promoter, and analyzed expression of the reporter using transient assay in Nicotiana

184 benthamiana. Three repeats provided the best expression of the reporter gene without 185 significant background (Fig 3A). The promoter architecture was further assayed for leaky 186 expression by generating pATF:YFP/BFP/RFP constructs and expressing gRNA 187 constitutively in the absence of dCas9:VP64 (Fig 3A). None of these constructs show 188 expression above background (Fig 3B and 3C). However, upon the addition of 189 constitutively expressed dCas9:VP64 cassette to the circuit, induction of reporter protein 190 expression was observed (Fig. 3B and 3C). Each pATF demonstrated comparable levels 191 of expression (pATF1:YFP - 3-fold, pATF3:BFP - 6-fold and pATF4:RFP - 2 fold) 192 compared to that obtained from the regular 35S promoter (6-fold; Fig 2B). The basic 193 features of the pATF and corresponding gRNAs can thus form the basis for the OCS and 194 should allow us to predictably control reporter and other gene circuits. The complete list 
195 of assembled OCS circuits is provided in Supplementary Table 2; as the reader will see,

196 OCS circuitry can be organized in terms of increasing complexity and demonstrates how

197 the Design-Built-Test approach can be used to empirically generate ever more

198 substantive plant phenotypes.

199 In order to show that the OCS designs could also function in stable transgenic Arabidopsis

200 thaliana lines, we assembled the OCS 1-1 and 4-1 circuits (Supplementary Table 2;

201 constitutive YFP and luciferase expression, respectively) in an Agrobacterium expression

202 vector containing with a kanamycin selectable marker as described previously. These

203 OCS constructs were successfully transformed into Arabidopsis thaliana plants (Fig 4A).

204 As expected, the OCS 1-1 $\mathrm{T}_{1}$ plants exhibited constitutive YFP expression (Fig 4B) while

205 the OCS 4-1 plants were imaged (as described in Methods) and the constitutive 206 expression of luciferase was confirmed (Fig 4C, 4D). Thus, the modular circuits 207 assembled function in two species, as infiltrates in Nicotiana and as transgenics in 208 Arabidopsis.

210 Inducible gene expression system via the OCS framework

212 The ability to precisely regulate the activity of the transgenes/circuit components based

213 on specific input stimuli is a key feature in programmable synthetic circuits $(48,49)$. In

214 order to enable orthogonal control of induction, we designed gRNA expression cassettes

215 to produce functional gRNAs from inducible Pol II promoters. To prevent nuclear export

216 of gRNAs due to capping and polyadenylation, we used the hammerhead ribozyme 217 (HHR) and Hepatitis Delta Virus (HDV) to cleave the 5' and the 3' ends of the gRNA, 
218 respectively. This strategy has been previously shown to lead to the expression of

219 functional gRNAs from Pol II promoters, with activity similar to those driven by the Pol III

220 (U6) promoter (50).

221

222 To proof the ribozyme processed gRNA constructs, OCS circuits were assembled where

223 gRNAs were either expressed from a U6 promoter (OCS 1-1) or the 35S promoter (OCS

224 1-5), and could subsequently activate the transcription and expression of reporter genes

225 (YFP) (Fig 5A). For both OCS circuits, downstream reporter gene expression was

226 observed, at similar levels (Fig. 5B). The specific levels of gRNA obtained in each case

227 were analyzed using qRT-PCR (Fig 5C and 5D), and as expected the level of gRNA from

228 the strong Pol II (35S) driven expression was higher than those obtained with the U6

229 promoter while similar levels of reporter expression were observed for both cases, thus

230 demonstrating that this Pol II driven gRNA expression strategy can be effectively used for

231 OCS activation (Fig 5E). For both these constructs the expression of hdCas9 (human

232 codon optimized dCas9) was also confirmed via Western blot analysis (Fig S2).

233 In order to demonstrate that the Pol II-driven gRNAs could be used as part of an inducible

234 OCS we used the well-characterized synthetic EBS promoter containing the EIN3 binding

235 (51), and placed YFP under the downstream control of the ATF (via pATF-1) (Fig 6A).

236 This circuit (OCS1-9) should be inducible by the volatile organic compound (VOC)

237 ethylene, which is produced from its precursor ACC (1-aminocyclopropane-1-carboxylic

238 acid). Time-dependent expression of YFP is observed in response to 10uM ACC

239 induction (Fig 6B). Both the gRNA-1 and YFP expression levels were quantified before

240 and after induction by qRT-PCR, a maximum of 3-fold induction was observed for both 
241 cases (Fig 6C and 6D). Thus, this demonstrates that the activity from synthetic promoters

242 can be controlled via the selective expression of the corresponding gRNAs.

244 Construction of a panel of mutually orthogonal synthetic promoters

246 Lack of multiplexed control of transgenes has been a major factor limiting the

247 development of synthetic circuits in plants $(5,6)$. Multiplexed regulation in turn requires

248 a panel of mutually orthogonal promoters and control elements that can operate

249 simultaneously $(5,6)$. Our strategy for synthetic promoter design naturally leads to the

250 generation of expression cassettes that are not only orthogonal to the host but are also

251 mutually orthogonal. The degree of orthogonality can be tuned at will via the sequence

252 design of the multiple gRNA components. By simply minimizing homology between

253 gRNAs, we constructed two additional promoters similar to the architecture of pATF-1, in

254 which gRNA binding sites were followed by a minimal 35S promoter (pATF-3 and pATF-

255 4). The orthogonality of these promoters was assayed by assembling expression

256 constructs in which each synthetic promoter controlled the production of a unique

257 fluorescent reporter (pATF-1: YFP, pATF-3: RFP and pATF-4: BFP). The respective

258 gRNAs (gRNA-1, gRNA-3 and gRNA-4) were separately transcribed from a U6 promoter

259 (Fig 7A). When expression constructs were infiltrated into Nicotiana benthamiana, each

260 of the synthetic promoters was specifically upregulated only when its corresponding

261 gRNA was expressed; no background was detected from the remaining two synthetic

262 promoters. (Fig 7B and 7C). 


\section{Construction of complex ratiometric circuits}

266 Now that we have a suite of mutually orthogonal promoters, we sought to construct simple

267 circuits where the activity of each promoter could be independently controlled. Three

268 separate reporter proteins were used to simultaneously monitor the activity of two

269 promoters: pATF-1 with YFP, while both RFP and BFP were under the control of the

270 pATF-3. By leveraging the designed, orthogonal behavior of these promoters it proved

271 possible to construct a ratiometric circuit wherein the activity of pATF-1, and hence YFP

272 expression, was under the control of ethylene (via ACC), while pATF-3 constitutively

273 drove the expression of RFP and BFP (Fig 8A). As expected, the addition of 10uM ACC,

274 induced the expression of YFP from the pATF-1 promoter (3-fold), while the expression

275 of the other reporters remained constant (Fig 8B and $\mathbf{8 C}$ ). The ratiometric response was

276 further validated by qRT-PCR; pATF-1 was induced 3-fold following a similar increase in

277 expression of gRNA-1 while there were no changes observed in the transcription of the

278 other two reporter genes (Fig $\mathbf{8 B}$ and $\mathbf{8 C}$ ). The predictable behavior of the designed,

279 artificial control elements in the ratiometric circuit is one of the first examples of complex

280 circuitry to be described in plants, and demonstrates uniquely how natural metabolism

281 and regulatory circuitry can be interfaced with free-standing orthogonal control systems.

\section{Discussion}


286 Transcriptional orthogonality is one of the bedrocks for circuit construction in synthetic

287 biology, and generally serves as the basis for the bottom-up construction of complex

288 circuitry for predictable dynamics $(7,10,17)$. For eukaryotes the construction of multiple

289 promoter elements is hindered by the typically complex regulatory sequences that lie

290 upstream and within promoters (52-54).

292 The design of synthetic eukaryotic promoters has traditionally implemented a common

293 architecture, where a strong transcriptional initiation region is cloned downstream of

294 orthogonal DNA binding operator sequences and the latter serve as landing pads for

295 synthetic transcription factors (23). The engineered transcription factors have typically

296 consisted of DNA binding proteins (i.e., prokaryotic DNA binding proteins like TetR, Lacl,

297 LexA and PhIF (55-57)) fused to well characterized transcriptional activation domain like

298 VP64. With the advent of programmable DNA binding proteins like zinc finger proteins 299 and TALEs the repertoire of synthetic promoters greatly increased $(23,24,58,59)$. That 300 said, each new synthetic promoter still requires the construction and characterization of 301 its own unique transcription factor $(23,59,60)$.

303 These bottlenecks can be circumvented by the use of the highly programmable RNA304 guided DNA binding protein dCas9 (26). The dCas9 RNP fused to transcription activation 305 domains such as VP64 has been used for the upregulation of endogenous genes in a 306 wide variety of eukaryotic species like yeast, mammalian cells and plants (16, 25, 26, 307 61). Here, we have used adapted this 'universal' transcription factor to control the 308 expression of synthetic and orthogonal promoters without the need of addition of any 
309 other factors. Using our modular framework, we were able to quickly design and

310 characterize a panel of mutually orthogonal promoters that could drive the production of

311 a variety of outputs, singly and in parallel, including different fluorescent proteins (GFP,

312 BFP, RFP and YFP) and luciferase.

314 The activities of dCas9 based transcription factors can potentially be controlled by simply 315 regulating the expression of their corresponding gRNAs $(16,17)$, enabling the coupling 316 of natural and synthetic transcription units, and thus natural and overlaid metabolic

317 responses. Here we have effectively used this strategy to couple ethylene sensing (via

318 known EIN3 binding sites) to synthetic (pATF) promoters. Moreover, by changing the 319 number and arrangement of gRNA binding sites synthetic promoters with different levels

320 of activation can be generated, providing further opportunities for design (62). While it

321 has been previously shown that a panel of minimal plant promoters can be used with

322 natural DNA binding sequences for modulating promoter strengths (20), the addition of

323 completely artificial, synthetic promoters as control elements should create opportunities

324 for increasing the specificity and strengths of engineered promoters.

325 Since our strategy for designing synthetic promoters is generalizable it is likely that even

326 more complex circuits can be built by simply incorporating other transcription factor

327 binding sites, or by changing the regulatory 'headpiece' on the dCas9 element (for

328 example, to a repressor), (63-65).

330 The stabilities of genetic circuitry in plants can be greatly modified by silencing and 331 recombination, amongst other mechanisms $(40,41,43)$. In this regard, the artificial 
332 promoter elements that we generate can potentially be crafted to avoid repetition (20),

333 and thus to better avoid silencing and recombination. As viable artificial promoter

334 sequences continue to accumulate, they can be compared and contrasted to identify

335 those that are least vulnerable to modification over time. The facile addition of new parts

336 to the standardize toolkit architecture, particularly terminators, will further increase

337 opportunities to avoid repetition in ways that again go well beyond what is possible by

338 relying on just a few well-characterized endogenous elements alone.

340 The implementation of orthogonal control systems in plants can be used to limit cross-

341 talk between natural and overlaid regulatory elements, allowing more precise response

342 to a variety of inputs, from VOCs to hormones to temperature, water, and nutrients. The

343 use of orthogonal control systems to enable more precise responses to pathogenesis is

344 especially intriguing given the presence of $\mathrm{R}$ genes that are specifically responsive to

345 individual pathogens (effector triggered immunity, ETI) (66). The architecture we have

346 developed is fully generalizable, and can potentially be expanded to non-model plants

347 and other eukaryotic species such as yeast and mammalian cells by the use of

348 appropriate transcription initiation regions under the control of similar gRNA sequences

349 binding sites (67).

351 Materials and Methods

352

353 Plasmid design and construction 
355 The plant expression vector was generated using the plasmid plCH86966

356 (Addgene\#48075) as the backbone. The lacZ expression cassette was replaced with the

357 GFP dropout sequence (Supplementary Table 2) to make the plasmid compatible with

358 YTK architecture design. All parts described in Supplementary Table 1, were cloned

359 into the backbone pYTK001 (Addgene \#65108). For the individual transcriptional units,

360 the backbone used was pYTK095 (Addgene \#65202) along with the appropriate

361 connector sequences described in Supplementary Table 3. For the design of orthogonal

362 gRNAs, random 20-mers were generated that had a GC content of $~ 50 \%$, and that were

363 at least 5 nucleotides away from all sequences in the Nicotiana and Arabidopsis

364 genomes. All oligonucleotides and gblocks were obtained from Integrated DNA

365 Technologies (IDT) unless otherwise stated.

366 For the construction of each genetic element namely promoters, coding sequences and

367 terminators, first they were checked for restriction sites for the following enzymes -

368 BsmBl, Bsal, Notl and Dralll. The restriction sites in the coding sequences were removed

369 by the use of synonymous codons while the other elements did not contain any of these

370 restriction sites. The complete list of parts and constructs are provided in Supplementary

371 Table 1. The part plasmids were cloned into a common vector where each genetic

372 element is flanked by Bsa1 restriction sites followed by appropriate overhangs

373 (Supplementary Table 1). For the assembly of both single TU or multi-TU, the following

374 procedure was used: $10 \mathrm{fmol}$ of backbone plasmid and $20 \mathrm{fmol}$ of parts/TUs were used

375 in a 10uL reaction with 1ul of 10x T4 ligase buffer along with 100 units of Bsal-v2 (single

376 TU) or Esp3I (multi-TU or parts) and 100 units of T7 DNA ligase. The cycling protocol

377 used is: 24 cycles of $3 \mathrm{~min}$ at $37^{\circ} \mathrm{C}$ (for digestion) and $5 \mathrm{~min}$ at $16^{\circ} \mathrm{C}$ (for ligation) followed 
378 by a final digestion step at $37^{\circ} \mathrm{C}$ for $30 \mathrm{~min}$ and the enzymes were heat inactivated $80^{\circ} \mathrm{C}$

379 for 20 min. All constructs were transformed into $\mathrm{DH} 10 \mathrm{~B}$ cells, grown at $37^{\circ} \mathrm{C}$ using 380 standard chemical transformation procedures. The colonies that lack fluorescence were

381 inoculated and plasmids were extracted using Qiagen Miniprep kit according to the 382 manufacturer's instructions Plasmids were maintained as the following antibiotics 383 kanamycin $(50 \mathrm{ug} / \mathrm{mL})$, chloramphenicol $(34 \mathrm{ug} / \mathrm{mL})$ and carbenicillin $(100 \mathrm{ug} / \mathrm{mL})$ 384 wherever required. The plasmids were sequence verified by Sanger sequencing (UT 385 Austin Genomic Sequencing and Analysis Facility). The correct constructs were then 386 transformed into Agrobacterium tumefaciens strain GV3101 (resistant to Gentamycin and 387 Rifampicin) and used either for transient expression in Nicotiana benthamiana or to 388 generate stable lines in Arabidopsis thaliana. The following enzymes were used for the 389 assemblies - Bsal-v2 (NEB \#R3733S), Esp3I (NEB \#R0734S) and T7 DNA ligase (NEB \#M0318S).

\section{Plant material, bacterial infiltration}

393 Nicotiana benthamiana and Arabidopsis thaliana plants were grown in soil at $22^{\circ} \mathrm{C}$, and $39416 \mathrm{hr}$ light period. For transient expression, three weeks old plants were syringe-infiltrated with Agrobacterium tumefaciens strain GV3101 $\left(\mathrm{OD}_{600}=0.5\right)$ and leaves were imaged

396 under Olympus BX53 Digital Fluorescence Microscope or harvested for RNA and/or 397 protein analysis. To create stable transformation in Arabidopsis, floral dip method (68)

398 was used. $T_{1}$ plants were selected on half MS Kanamycin $(50 \mu \mathrm{g} / \mathrm{ml})$ plates and the 399 selected T1 plants were analyzed using an Olympus BX53 Digital Fluorescence 400 Microscope and a NightOwl imager for YFP expression and luciferase expression, 
401 respectively. For circuits that constitutively expressed YFP (OCS1-1) and luciferase

402 (OCS4-1) no other obvious phenotypic differences were observed across numerous

403 individual plants.

404

$405 \quad$ RNA extraction and qRT-PCR

406 RNA was extracted using TRIzol reagent (Ambion). $1 \mu \mathrm{g}$ total RNA was used to synthesize

407 cDNA. After DNasel treatment to remove any DNA contamination, random primer mix 408 (NEB \#S1330S) and M-MLV Reverse transcriptase (Invitrogen \#28025-013) were used

409 for first strand synthesis. qRT-PCR was used to quantify the RNA prepared from transient 410 expression experiments. AzuraQuant qPCR Master Mix (Azura Genomics) was used with

411 initial incubation at $95^{\circ} \mathrm{C}$ for 2 min followed by 40 cycles of $95^{\circ} \mathrm{C}$ for 10 sec and $60{ }^{\circ} \mathrm{C}$

412 for $30 \mathrm{sec}$. Level of target RNA was calculated from the difference of threshold cycle (Ct)

413 values between reference (5S rRNA) and target gene using at least three independent 414 replicates

\section{ACC treatment}

417 To check the induction of reporter in response to ACC in the plasmids containing 418 pEBS::YFP/RFP/BFP, Nicotiana benthamiana leaves were infiltrated with Agrobacterium;

419 after three days post infiltration, leaf discs were cut using cork borer and incubated in 420 either $0 \mu \mathrm{M}$ or $10 \mu \mathrm{M}$ ACC for four hours. Fluorescence microscopy was used to check 421 YFP expression after induction.

422

423 Fluorescence and Luminescence imaging 
424 Fluorescence microscope images after Agrobacterium mediated transient expression of

425 YFP, BFP, RFP and GFP in Nicotiana benthamiana leaves were taken using an Olympus

426 BX53 Digital Fluorescence Microscope. For this purpose, leaf discs were cut using cork

427 borer from the area which was infiltrated. Images were taken using either 10X objective

428 lens using the default filters for YFP (500/535nm), BFP (385/448nm), and RFP

429 (560/630nm). The UV filter (350/460nm) was used to take GFP images. The exposure

430 and gain setting were kept constant for each filter within each experiment to compare

431 multiple leaf discs (3 to 6). In all the experiments a leaf disc from a leaf which was not

432 infiltrated with Agrobacterium was used as a negative control in order to account for

433 background fluorescence. All experiments were performed at least three times

434 independently as indicated in the Results.

435 Expression of luciferase was detected using NightOwl II LB 983 in vivo imaging system

436 (https://www.berthold.com/en/bioanalytic/products/in-vivo-imaging-systems/nightowl-

$437 \mathrm{lb} 983 /)$. Leaves/plants were sprayed with $100 \mu \mathrm{M}$ D-luciferin, Potassium salt (GoldBio

438 \#LUCK-300). After 5 min of incubation, images were taken in the NightOwl II LB 983.

439 Images were captured with a backlit NightOWL LB 983 NC 100 CCD camera. Photons

440 emitted from luciferase were collected and integrated for a 2 min period. A pseudocolor

441 luminescent image from blue (least intense) to red (most intense), representing the

442 distribution of the detected photons emitted from active luciferase was generated using

443 Indigo software (Berthold Technologies).

444

445 Western blot

446 Total protein was extracted using urea-based denaturing buffer (100 mM NaH2PO4, 8 M 
447 urea, and $10 \mathrm{mM}$ Tris- $\mathrm{HCl}, \mathrm{pH}$ 8.0) and used for immunoblot analysis to check the 448 expression. The proteins were fractionated by $8 \%$ SDS-PAGE gel and transferred to a 449 polyvinylidene difluoride (PVDF) membrane using a transfer apparatus according to the 450 manufacturer's protocols (Bio-Rad). The membrane was treated with 5\% nonfat milk in 451 PBS-T for 10 min for blocking, and then incubated with Cas9 antibody (Santa cruz, 7A9$4523 \mathrm{~A} 3,1: 500)$ at $4{ }^{\circ} \mathrm{C}$ for overnight. After incubation, the membrane was washed three times 453 for $5 \mathrm{~min}$ and incubated with horseradish peroxidase-conjugated anti-mouse (1:10000)

454 for $2 \mathrm{~h}$. The Blot was washed with PBS-T three times and detected with the ECL system 455 (Thermo scientific, lot\# SE251206).

456

457 Declarations

458

459 Ethics approval and consent to participate

460 Not Applicable

461

462 Consent for publication

463 Not Applicable

465 Availability of data and materials

466

467 Competing interests

468 The authors declare no competing interests. 
$470 \quad$ Funding

471 This work was supported by the Defense Advanced Research Projects Agency (DARPA)

472 agreement HR00111820048 to AE and SS. The content of the information does not

473 necessarily reflect the position or the policy of the Government, and no official

474 endorsement should be inferred. This work was also supported by Welch Foundation

475 grant (F-1654) to ADE.

476

477 Author' contributions

478 SK, SS and AE conceived of the project. SK designed the framework and the basic

479 elements of OCS with input from EG, JG and SS. SK and YB assembled all constructs.

$480 \mathrm{YB}, \mathrm{NR}$ and JK performed all the testing in Nicotiana with input from SS. All authors

481 contributed with the preparation of figures. SK, YB, JK, SS and AE wrote the manuscript

482 with input from all authors.

483

484 Acknowledgments

485 We would also like to thank the Qiao lab (UT Austin) for providing details regarding the 486 ethylene induction of the OCS constructs.

Supplementary Information includes

Fig S1: Workflow describing the assembly of single and multiple transcriptional units (TUs) in a plant expression vector; Fig S2: Western blot to analyze the expression of dCas9:VP64 in OCS constructs - OCS1-1 and OCS 1-5

Table S1: List of all genetic parts used for the construction of OCS constructs

Table S2: List of all OCS constructs

Table S3: List of all Addgene plasmids used in this work

Full OCS plasmid maps 


\section{References}

1. Tolle F, Stucheli P, Fussenegger M. Genetic circuitry for personalized human cell therapy. Current opinion in biotechnology. 2019;59:31-8.

503 2. Scheller L, Fussenegger M. From synthetic biology to human therapy: engineered mammalian cells. Current opinion in biotechnology. 2019;58:108-16.

505 3. Sedlmayer F, Aubel D, Fussenegger M. Synthetic gene circuits for the detection, 506 elimination and prevention of disease. Nature biomedical engineering. 2018;2(6):399-415.

507 4. Riglar DT, Silver PA. Engineering bacteria for diagnostic and therapeutic applications. 508 Nature reviews Microbiology. 2018;16(4):214-25.

$509 \quad 5 . \quad$ Kassaw TK, Donayre-Torres AJ, Antunes MS, Morey KJ, Medford JI. Engineering synthetic 510 regulatory circuits in plants. Plant science : an international journal of experimental plant biology. $5112018 ; 273: 13-22$.

512 6. de Lange O, Klavins E, Nemhauser J. Synthetic genetic circuits in crop plants. Current 513 opinion in biotechnology. 2018;49:16-22.

514 7. Nielsen AA, Der BS, Shin J, Vaidyanathan P, Paralanov V, Strychalski EA, et al. Genetic 515 circuit design automation. Science (New York, NY). 2016;352(6281):aac7341.

516 8. Medford JI, Prasad A. Towards programmable plant genetic circuits. The Plant journal : 517 for cell and molecular biology. 2016;87(1):139-48.

518 9. Fonseca JP, Bonny AR, Kumar GR, Ng AH, Town J, Wu QC, et al. A Toolkit for Rapid Modular 519 Construction of Biological Circuits in Mammalian Cells. ACS synthetic biology. 2019;8(11):2593520606.

521 10. Brophy JA, Voigt CA. Principles of genetic circuit design. Nature methods. 2014;11(5):508-

52220.

523 11. Mutalik VK, Guimaraes JC, Cambray G, Lam C, Christoffersen MJ, Mai QA, et al. Precise 524 and reliable gene expression via standard transcription and translation initiation elements. 525 Nature methods. 2013;10(4):354-60.

526 12. Weinberg BH, Pham NTH, Caraballo LD, Lozanoski T, Engel A, Bhatia S, et al. Large-scale 527 design of robust genetic circuits with multiple inputs and outputs for mammalian cells. Nature 528 biotechnology. 2017;35(5):453-62.

529 13. Basu S, Gerchman Y, Collins CH, Arnold FH, Weiss R. A synthetic multicellular system for 530 programmed pattern formation. Nature. 2005;434(7037):1130-4.

531 14. Gardner TS, Cantor CR, Collins JJ. Construction of a genetic toggle switch in Escherichia 532 coli. Nature. 2000;403(6767):339-42.

533 15. Elowitz MB, Leibler S. A synthetic oscillatory network of transcriptional regulators. 534 Nature. 2000;403(6767):335-8.

535 16. Kim H, Bojar D, Fussenegger M. A CRISPR/Cas9-based central processing unit to program 536 complex logic computation in human cells. Proceedings of the National Academy of Sciences of 537 the United States of America. 2019;116(15):7214-9.

538 17. Gander MW, Vrana JD, Voje WE, Carothers JM, Klavins E. Digital logic circuits in yeast with 539 CRISPR-dCas9 NOR gates. Nature communications. 2017;8:15459.

540 18. Weber W, Fussenegger M. Engineering of synthetic mammalian gene networks. 541 Chemistry \& biology. 2009;16(3):287-97. 
542 19. Kramer BP, Fussenegger M. Hysteresis in a synthetic mammalian gene network. 543 Proceedings of the National Academy of Sciences of the United States of America. $5442005 ; 102(27): 9517-22$.

545 20. Belcher MS, Vuu KM, Zhou A, Mansoori N, Agosto Ramos A, Thompson MG, et al. Design 546 of orthogonal regulatory systems for modulating gene expression in plants. Nature chemical 547 biology. 2020;16(8):857-65.

548 21. Schaumberg KA, Antunes MS, Kassaw TK, Xu W, Zalewski CS, Medford Jl, et al. 549 Quantitative characterization of genetic parts and circuits for plant synthetic biology. Nature 550 methods. 2016;13(1):94-100.

551 22. Brückner K, Schäfer P, Weber E, Grützner R, Marillonnet S, Tissier A. A library of synthetic 552 transcription activator-like effector-activated promoters for coordinated orthogonal 553 gene expression in plants. The Plant journal : for cell and molecular biology. 2015;82(4):707-16. 554 23. Khalil AS, Lu TK, Bashor CJ, Ramirez CL, Pyenson NC, Joung JK, et al. A synthetic biology 555 framework for programming eukaryotic transcription functions. Cell. 2012;150(3):647-58. 24. Bae KH, Kwon YD, Shin HC, Hwang MS, Ryu EH, Park KS, et al. Human zinc fingers as building blocks in the construction of artificial transcription factors. Nature biotechnology. 2003;21(3):275-80.

560 for plant and mammalian cells. Nature plants. 2017;3(12):930-6.

561 26. Perez-Pinera P, Kocak DD, Vockley CM, Adler AF, Kabadi AM, Polstein LR, et al. RNA-guided 562 gene activation by CRISPR-Cas9-based transcription factors. Nature methods. 2013;10(10):9735636.

564 27. Chavez A, Tuttle M, Pruitt BW, Ewen-Campen B, Chari R, Ter-Ovanesyan D, et al. Comparison of Cas9 activators in multiple species. Nature methods. 2016;13(7):563-7.

566 28. Lee ME, DeLoache WC, Cervantes B, Dueber JE. A Highly Characterized Yeast Toolkit for 567 Modular, Multipart Assembly. ACS synthetic biology. 2015;4(9):975-86.

568 29. Engler C, Youles M, Gruetzner R, Ehnert TM, Werner S, Jones JD, et al. A golden gate 569 modular cloning toolbox for plants. ACS synthetic biology. 2014;3(11):839-43.

570 30. Weber E, Engler C, Gruetzner R, Werner S, Marillonnet S. A modular cloning system for 571 standardized assembly of multigene constructs. PloS one. 2011;6(2):e16765.

572 31. Banta LM, Montenegro M. Agrobacterium and Plant Biotechnology. In: Tzfira T, Citovsky 573 V, editors. Agrobacterium: From Biology to Biotechnology. New York, NY: Springer New York; 574 2008. p. 73-147.

575 32. Berg RH, Beachy RN. Fluorescent protein applications in plants. Methods Cell Biol. $576 \quad 2008 ; 85: 153-77$.

577 33. Benfey PN, Chua NH. The Cauliflower Mosaic Virus 35S Promoter: Combinatorial 578 Regulation of Transcription in Plants. Science (New York, NY). 1990;250(4983):959-66.

579 34. Odell JT, Nagy F, Chua NH. Identification of DNA sequences required for activity of the 580 cauliflower mosaic virus 35S promoter. Nature. 1985;313(6005):810-2.

$581 \quad 35 . \quad K e r p p o l a ~ T K$. Bimolecular Fluorescence Complementation (BiFC) Analysis as a Probe of 582 Protein Interactions in Living Cells. Annual Review of Biophysics. 2008;37(1):465-87.

583 36. Tindall AJ, Waller J, Greenwood M, Gould PD, Hartwell J, Hall A. A comparison of high584 throughput techniques for assaying circadian rhythms in plants. Plant Methods. 2015;11(1):32. 
37. Xiong TC, Sanchez F, Briat J-F, Gaymard F, Dubos C. Spatio-Temporal Imaging of Promoter Activity in Intact Plant Tissues. In: Hehl R, editor. Plant Synthetic Promoters: Methods and Protocols. New York, NY: Springer New York; 2016. p. 103-10.

38. Xu G, Greene GH, Yoo H, Liu L, Marqués J, Motley J, et al. Global translational reprogramming is a fundamental layer of immune regulation in plants. Nature. 2017;545(7655):487-90.

39. Zhou M, Wang W, Karapetyan S, Mwimba M, Marqués J, Buchler NE, et al. Redox rhythm reinforces the circadian clock to gate immune response. Nature. 2015;523:472.

40. Vaillant I, Schubert I, Tourmente S, Mathieu O. MOM1 mediates DNA-methylationindependent silencing of repetitive sequences in Arabidopsis. EMBO Rep. 2006;7(12):1273-8.

41. Matzke MA, Mette MF, Matzke AJ. Transgene silencing by the host genome defense: implications for the evolution of epigenetic control mechanisms in plants and vertebrates. Plant Mol Biol. 2000;43(2-3):401-15.

42. Ni M, Cui D, Einstein J, Narasimhulu S, Vergara CE, Gelvin SB. Strength and tissue specificity of chimeric promoters derived from the octopine and mannopine synthase genes. The Plant Journal. 1995;7(4):661-76.

43. Matzke MA, Primig M, Trnovsky J, Matzke AJ. Reversible methylation and inactivation of marker genes in sequentially transformed tobacco plants. Embo j. 1989;8(3):643-9.

44. Cardinale S, Arkin AP. Contextualizing context for synthetic biology - identifying causes of failure of synthetic biological systems. Biotechnology Journal. 2012;7(7):856-66.

45. Kang H-G, Fang Y, Singh KB. A glucocorticoid-inducible transcription system causes severe growth defects in Arabidopsis and induces defense-related genes. The Plant Journal. 1999;20(1):127-33.

46. Amirsadeghi $S$, McDonald AE, Vanlerberghe GC. A glucocorticoid-inducible gene expression system can cause growth defects in tobacco. Planta. 2007;226(2):453-63.

47. Lowder LG, Paul JW, 3rd, Qi Y. Multiplexed Transcriptional Activation or Repression in Plants Using CRISPR-dCas9-Based Systems. Methods in molecular biology (Clifton, NJ). 2017;1629:167-84.

48. Bayer TS, Smolke CD. Programmable ligand-controlled riboregulators of eukaryotic gene expression. Nature Biotechnology. 2005;23(3):337-43.

49. Kotula JW, Kerns SJ, Shaket LA, Siraj L, Collins JJ, Way JC, et al. Programmable bacteria detect and record an environmental signal in the mammalian gut. Proceedings of the National Academy of Sciences. 2014;111(13):4838.

50. Jacobs JZ, Ciccaglione KM, Tournier V, Zaratiegui M. Implementation of the CRISPR-Cas9 system in fission yeast. Nature Communications. 2014;5(1):5344.

51. Cruz AB, Bianchetti RE, Alves FRR, Purgatto E, Peres LEP, Rossi M, et al. Light, Ethylene and Auxin Signaling Interaction Regulates Carotenoid Biosynthesis During Tomato Fruit Ripening. Frontiers in Plant Science. 2018;9(1370).

52. Lelli KM, Slattery M, Mann RS. Disentangling the many layers of eukaryotic transcriptional regulation. Annual review of genetics. 2012;46:43-68.

53. Ellwood K, Huang W, Johnson R, Carey M. Multiple layers of cooperativity regulate enhanceosome-responsive RNA polymerase II transcription complex assembly. Mol Cell Biol. 1999;19(4):2613-23. 
628 54. Chen L. Combinatorial gene regulation by eukaryotic transcription factors. Curr Opin Struct Biol. 1999;9(1):48-55.

630 55. Urlinger S, Baron U, Thellmann M, Hasan MT, Bujard H, Hillen W. Exploring the sequence space for tetracycline-dependent transcriptional activators: novel mutations yield expanded range and sensitivity. Proceedings of the National Academy of Sciences of the United States of America. 2000;97(14):7963-8.

634 56. Bellí G, Garí E, Piedrafita L, Aldea M, Herrero E. An activator/repressor dual system allows tight tetracycline-regulated gene expression in budding yeast. Nucleic Acids Research. 1998;26(4):942-7.

637 57. Gossen M, Bujard H. Tight control of gene expression in mammalian cells by tetracyclineresponsive promoters. Proceedings of the National Academy of Sciences of the United States of America. 1992;89(12):5547-51.

640 58. Perez-Pinera P, Ousterout DG, Brunger JM, Farin AM, Glass KA, Guilak F, et al. Synergistic 641 and tunable human gene activation by combinations of synthetic transcription factors. Nature 642 methods. 2013;10(3):239-42.

643 59. Li Y, Moore R, Guinn M, Bleris L. Transcription activator-like effector hybrids for conditional control and rewiring of chromosomal transgene expression. Sci Rep. 2012;2:897. 60. Cermak T, Doyle EL, Christian M, Wang L, Zhang Y, Schmidt C, et al. Efficient design and assembly of custom TALEN and other TAL effector-based constructs for DNA targeting. Nucleic Acids Research. 2011;39(12):e82.

648 61. Lowder LG, Zhang D, Baltes NJ, Paul JW, 3rd, Tang X, Zheng X, et al. A CRISPR/Cas9 Toolbox 649 for Multiplexed Plant Genome Editing and Transcriptional Regulation. Plant physiology. $650 \quad 2015 ; 169(2): 971-85$.

651 62. Kocak DD, Josephs EA, Bhandarkar V, Adkar SS, Kwon JB, Gersbach CA. Increasing the 652 specificity of CRISPR systems with engineered RNA secondary structures. Nature biotechnology. 653 2019;37(6):657-66.

654 63. Pandelakis M, Delgado E, Ebrahimkhani MR. CRISPR-Based Synthetic Transcription 655 Factors In Vivo: The Future of Therapeutic Cellular Programming. Cell Syst. 2020;10(1):1-14.

656 64. Yeo NC, Chavez A, Lance-Byrne A, Chan Y, Menn D, Milanova D, et al. An enhanced CRISPR 657 repressor for targeted mammalian gene regulation. Nature methods. 2018;15(8):611-6.

658 65. Kwon DY, Zhao YT, Lamonica JM, Zhou Z. Locus-specific histone deacetylation using a 659 synthetic CRISPR-Cas9-based HDAC. Nature communications. 2017;8:15315.

660 66. Gonzalez TL, Liang Y, Nguyen BN, Staskawicz BJ, Loqué D, Hammond MC. Tight regulation 661 of plant immune responses by combining promoter and suicide exon elements. Nucleic Acids 662 Research. 2015;43(14):7152-61.

663 67. Farzadfard F, Perli SD, Lu TK. Tunable and multifunctional eukaryotic transcription factors 664 based on CRISPR/Cas. ACS synthetic biology. 2013;2(10):604-13.

665 68. Zhang X, Henriques R, Lin S-S, Niu Q-W, Chua N-H. Agrobacterium-mediated 666 transformation of Arabidopsis thaliana using the floral dip method. Nature Protocols. 667 2006;1(2):641-6. 
A

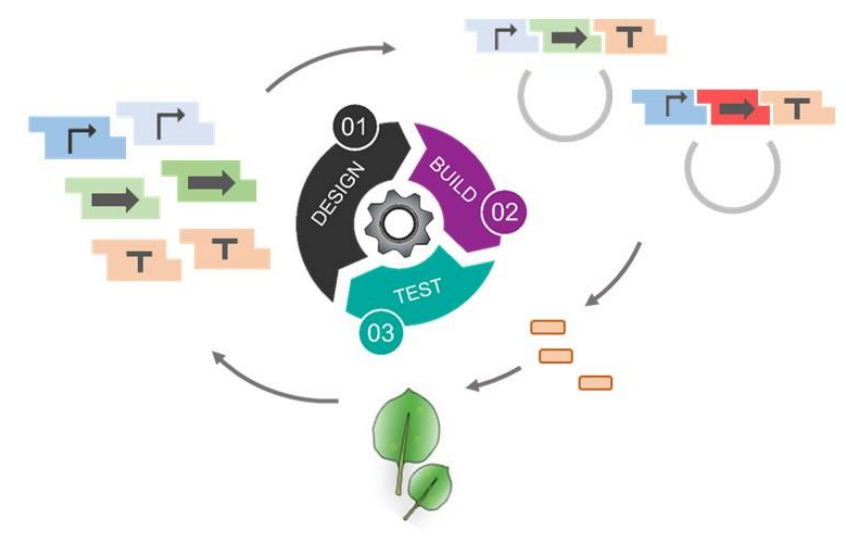

B

Plant Resistance

pVS1

Bacterial KanR

pBR322

Figure 1. Schematic overview of the design-build-test cycle A. Genetic elements such as promoters, genes and terminators are encoded as modular parts consisting of Bsal recognition sites flanked by specific overhangs to ensure the hierarchical assembly of transcriptional units. Once assembled, the constructs are transformed into Agrobacterium and the reporter expression is characterized in Nicotiana benthamiana leaf infiltrates B. Design of the shuttle vector backbone used for the assembly of 
A

A Non-infiltrated
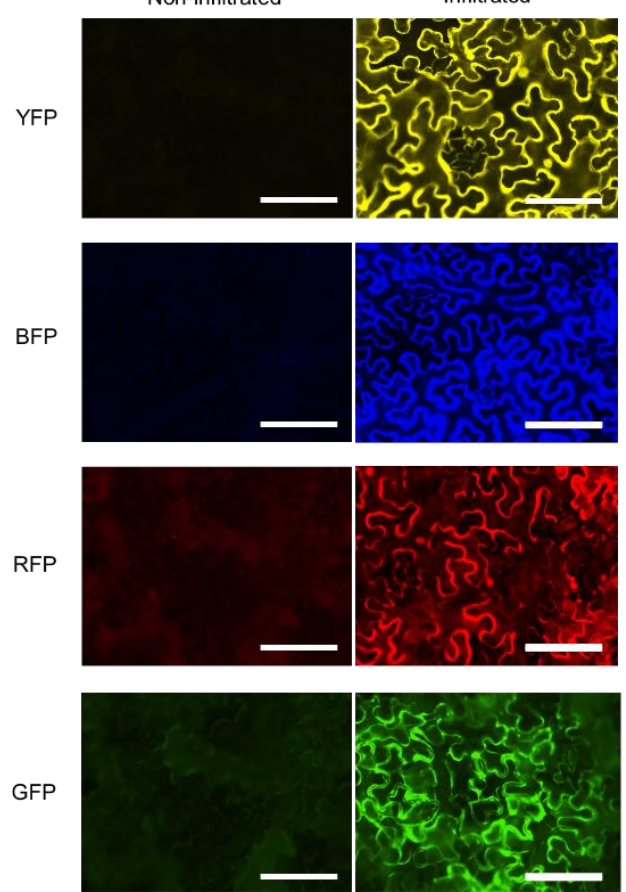

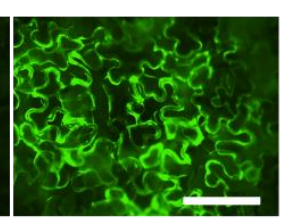

Scale bar : $200 \mu \mathrm{m}$

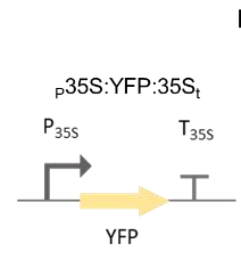

p35S:BFP:35S

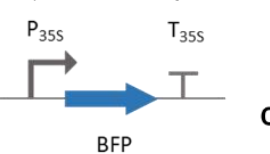

p35S:RFP:35S

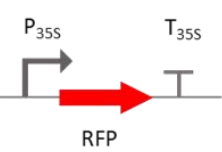

p35S:sfGFP:35S

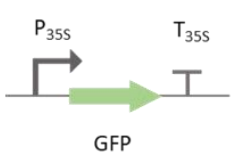

GFP

B

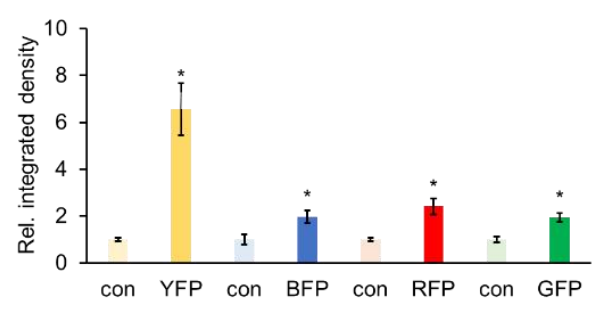

c

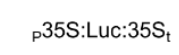

D

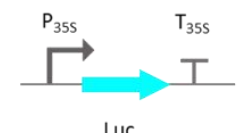

Luc

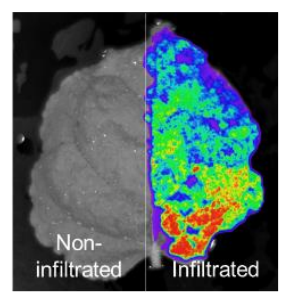

YFP
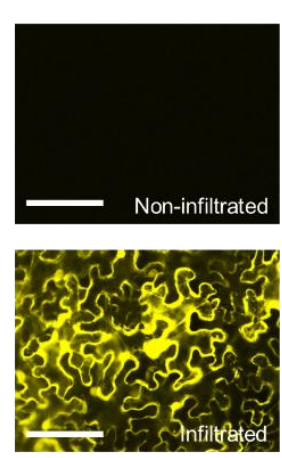

YFP
680

681

682

683

684

685

686

687

688

689

690

691

692

693

694

695

Figure 2. Characterization of reporter constructs assembled using APT toolkit. A. Fluorescence microscope images showing Agrobacterium mediated transient expression of YFP, BFP, RFP and GFP under the control of $35 \mathrm{~S}$ promoter into Nicotiana benthamiana leaves. Images on the left are from non-infiltrated leaves (negative control) captured using the appropriate filter at same exposure and gain settings as was used for the positive images on the right (Material and Methods). B. Relative integrated density of each fluorescence signal (shown in panel A). Integrated density was measured using image $\mathrm{J}$ software and normalized to that of a non-infiltrated control (con). Error bars: S.D. $(n=3$, independent replicates). Asterisks indicate statistical significance in a student t-test $(\mathrm{P}<0.05)$. C. Luminescence reporter luciferase expression shown by Agrobacterium mediated transient expression of luciferase in Nicotiana benthamiana leaves. Left half of the leaf was not infiltrated with Agrobacterium. D. Fluorescence microscope images showing Agrobacterium mediated transient expression of YFP under MAS promoter in Nicotiana benthamiana leaves. Image on the left is the brightfield image for the same construct. 
A

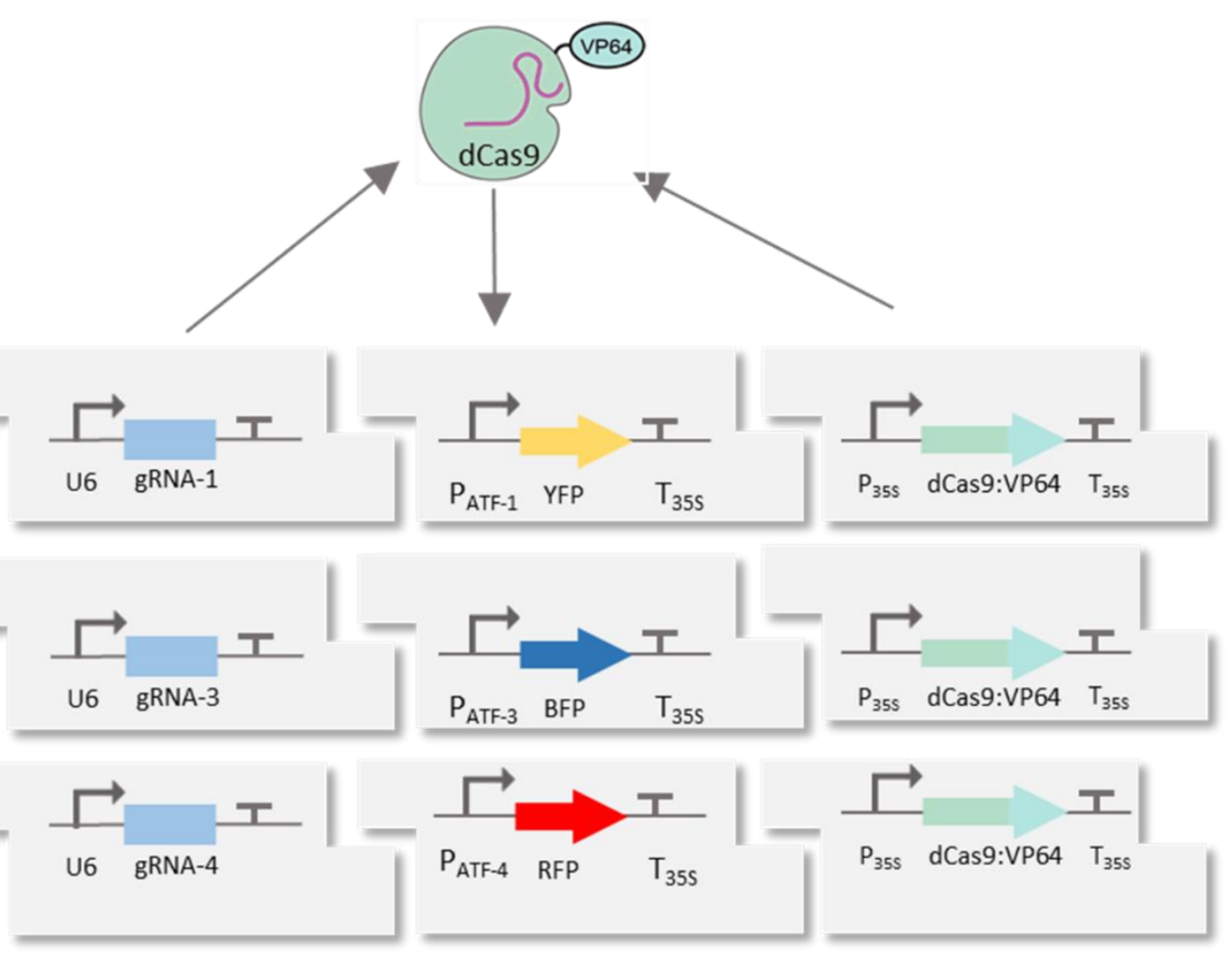

B
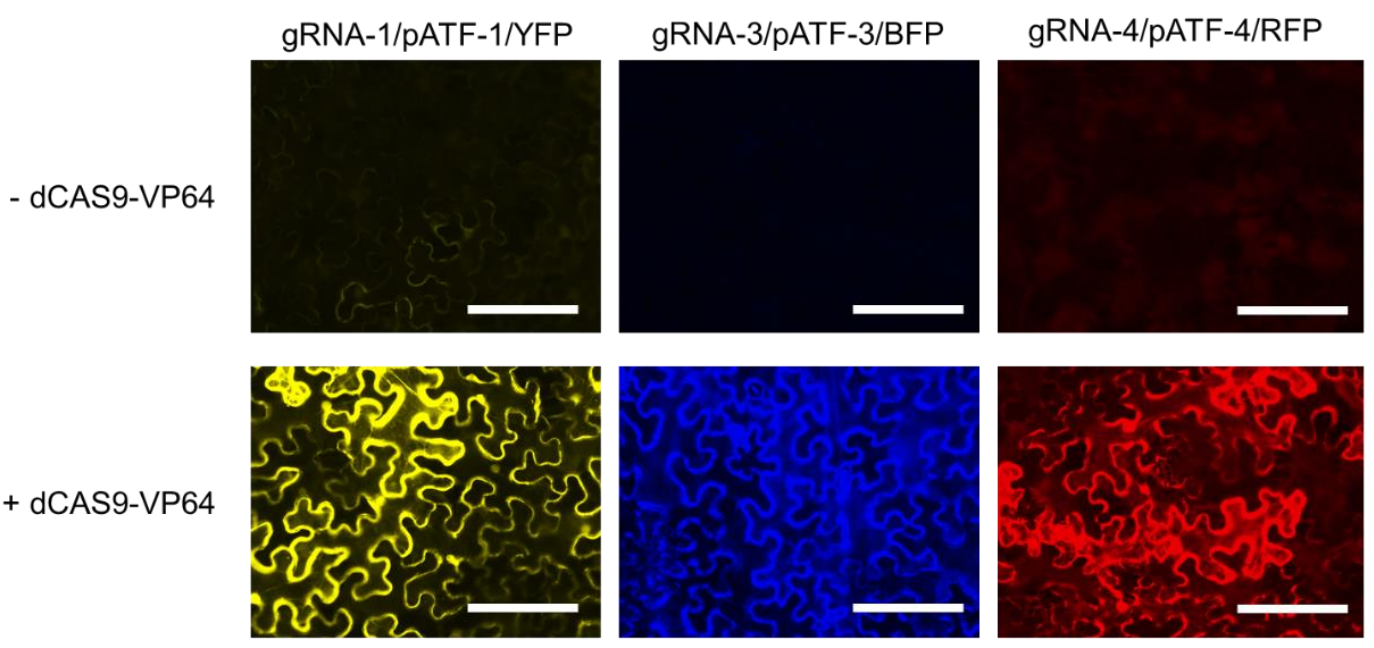

C
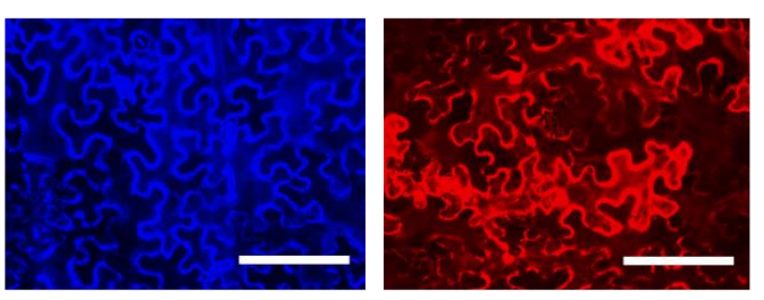

Scale bar : $200 \mu \mathrm{m}$
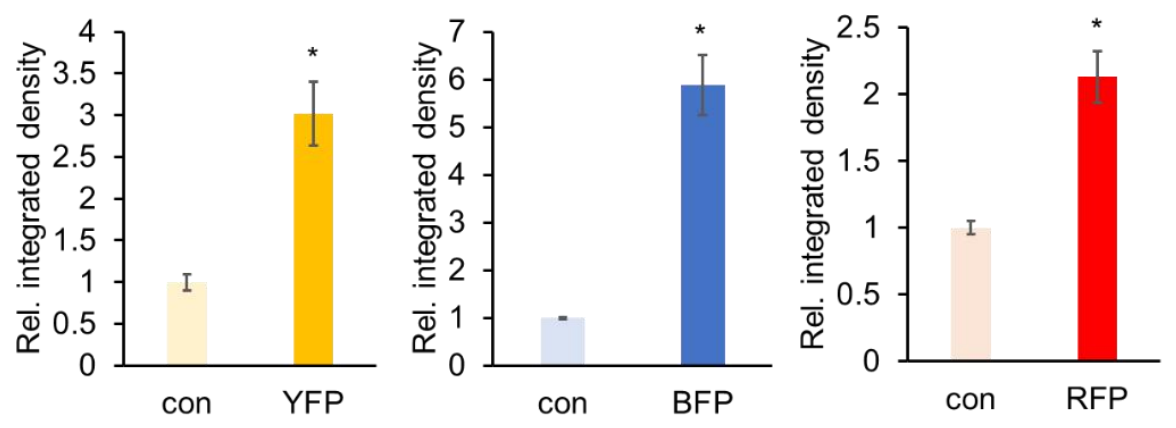
697 Figure 3. Characterization of activity of synthetic pATF promoters. A. Circuit design 698 of dCas9 based artificial transcription factor-controlled activation of synthetic promoters 699 (pATFs). Specific gRNAs are produced by U6 promoter while the expression of the 700 dCas9-VP64 is under the control of the 35S promoter. Reporter genes are under the 701 control of the synthetic promoter (3 repeats of the gRNA followed by minimal 35S 702 promoter to the artificial promoter ( $g R N A$ binding site) upstream of a specific fluorescence 703 reporter. B. Fluorescence microscope image showing Agrobacterium mediated transient 704 expression of YFP, BFP and RFP into Nicotiana benthamiana leaves with dCas9-VP64 705 (bottom panels) and without dCas9-VP64 (upper panels) using three different gRNAs. 706 Images were captured using the appropriate filter (Materials and Methods) at same 707 exposure. C. Relative integrated density of each fluorescence signal (shown in panel B). 708 Integrated density was measured using image $\mathrm{J}$ software and normalized to that of the 709 control (con; - dCAS9-VP64). Error bars: S.D. ( $n=3$, independent replicates). Asterisks 710 indicate statistical significance in a student $t$-test $(P<0.05)$. 
A

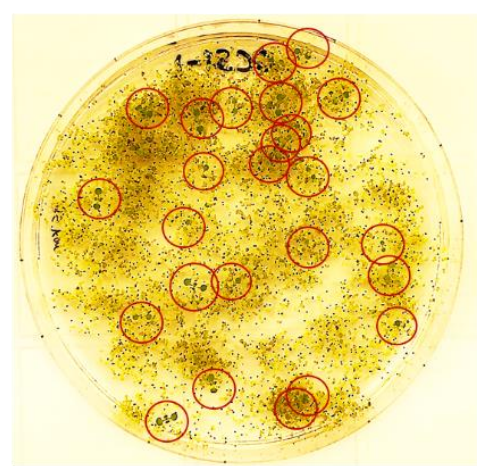

C

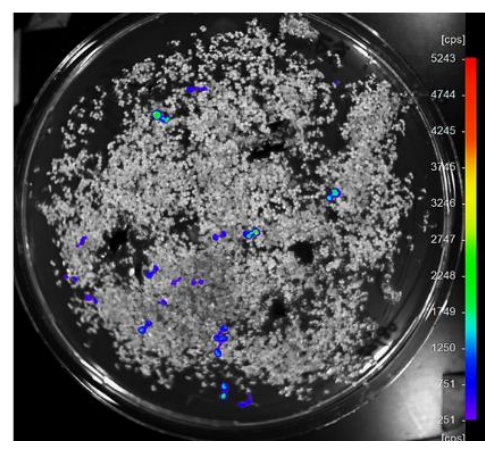

B

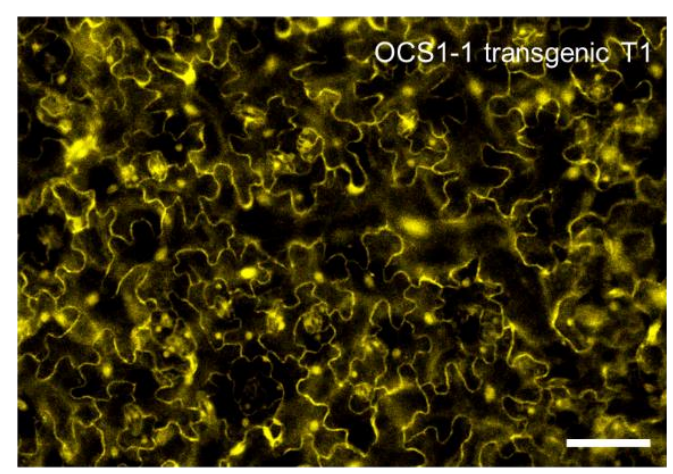

D

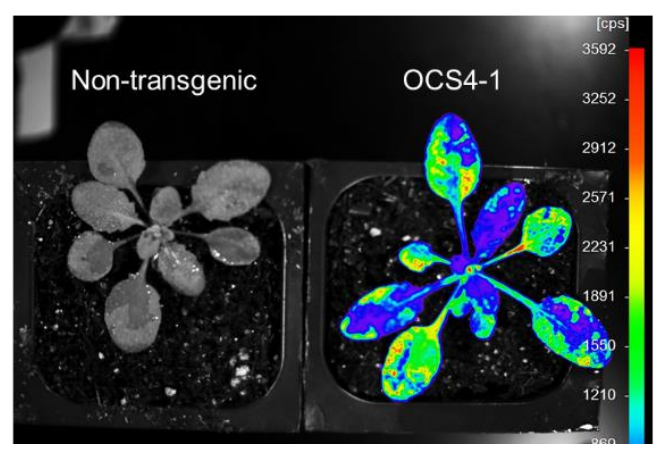

Figure 4. Evaluation of OCS reporter gene expression in transgenic Arabidopsis plants. A. Image showing Kanamycin selection of the transgenic Arabidopsis seedlings on MS media. Seedlings highlighted in the red circle have successfully incorporated OCS circuit. Transformation efficiency is within reasonable ranges $(\sim 1 \%)$ determined by a simple evaluation of the identified seedlings. B. Fluorescence microscope image of

717 Arabidopsis transgenic $\mathrm{T}_{1}$ plants containing the constitutive expression of YFP under the

718 OCS control (OCS 1-1). Scale bar: $50 \mu \mathrm{m}$ C. Image showing Kanamycin selection of the 719 transgenic Arabidopsis seedlings on MS media using luminescence reporter (OCS4-1) 720 taken using the NightOwl (Methods). D. Image of a T 1 Arabidopsis plant containing OCS47211 at the rosette stage after spraying the luciferin (Methods) containing OCS4-1. This 722 image, taken at the rosette stage using NightOwl after luciferin spray, shows that the 723 luciferase expression is active throughout the adult plant. A non-transgenic plant on the left was used as a negative control in the luminescence reporter assay. 


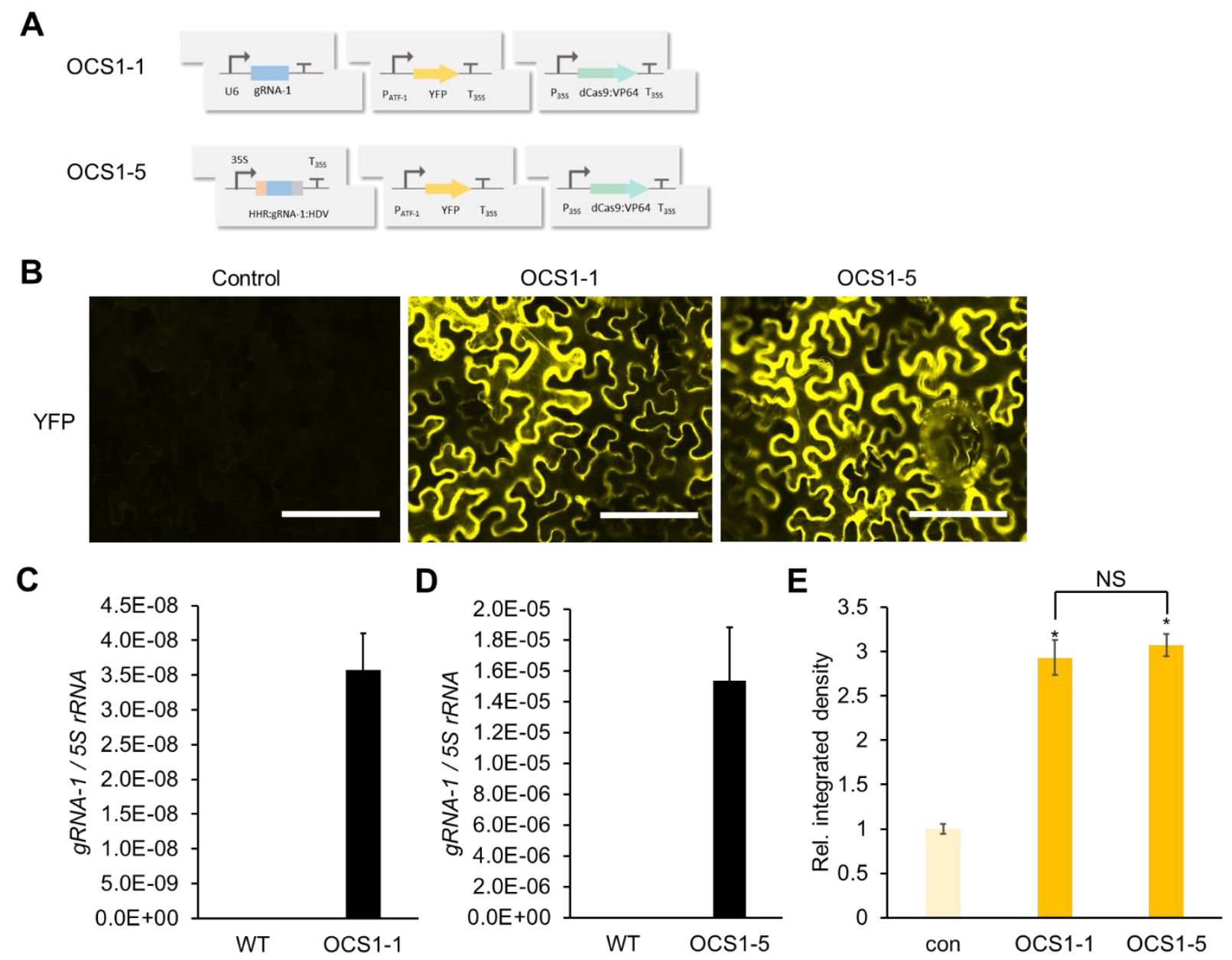
control of Pol II promoters. A. OCS1-1 circuit generates RNA using U6 (Pol III) promoter while OCS1-5 circuit generates gRNA using 35S (Pol II) promoter flanked by self-cleaving ribozymes - HammerHead (HHR) and Hepatitis Delta Virus (HDV). B. Fluorescence microscope images showing Agrobacterium mediated transient expression of OCS constructs with two modalities of gRNA expression (OCS1-1 and OCS1-5). Control 732 images were taken without dCAS9-VP64 expression. Scale bars: $200 \mu \mathrm{m}$ C and D. 733 Quantification of the gRNA-1 expression in OCS constructs (OCS 1-1 (C) and OCS 1-5 (D)) using qPCR relative to $5 S$ rRNA. Error bars : S.D. ( $n=3$, independent replicates) $E$. Relative integrated density of each fluorescence signal (shown in panel B). Integrated density was measured using image $\mathrm{J}$ software and normalized to that of the control (con; - dCas9-VP64). Error bars: S.D. ( $n=3$, independent replicates). Asterisks indicate statistical significance in a student t-test $(P<0.05)$. NS: not significant. 
A

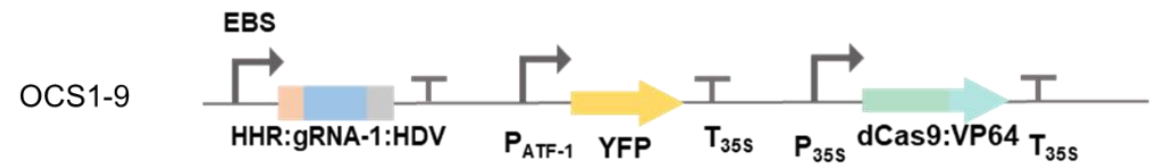

B
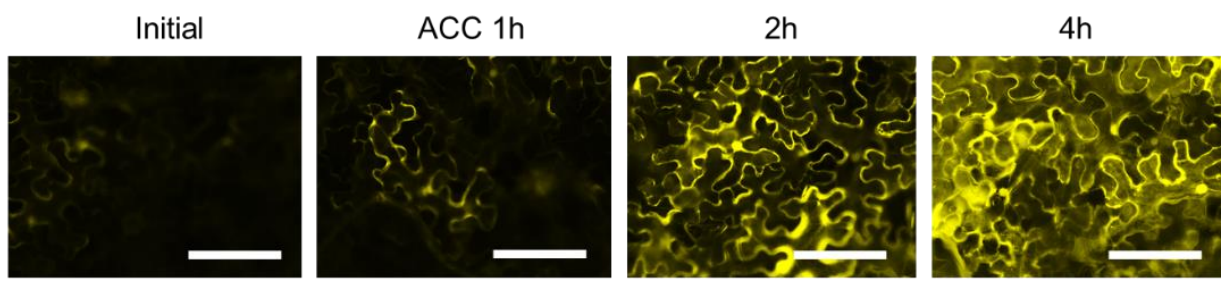

C
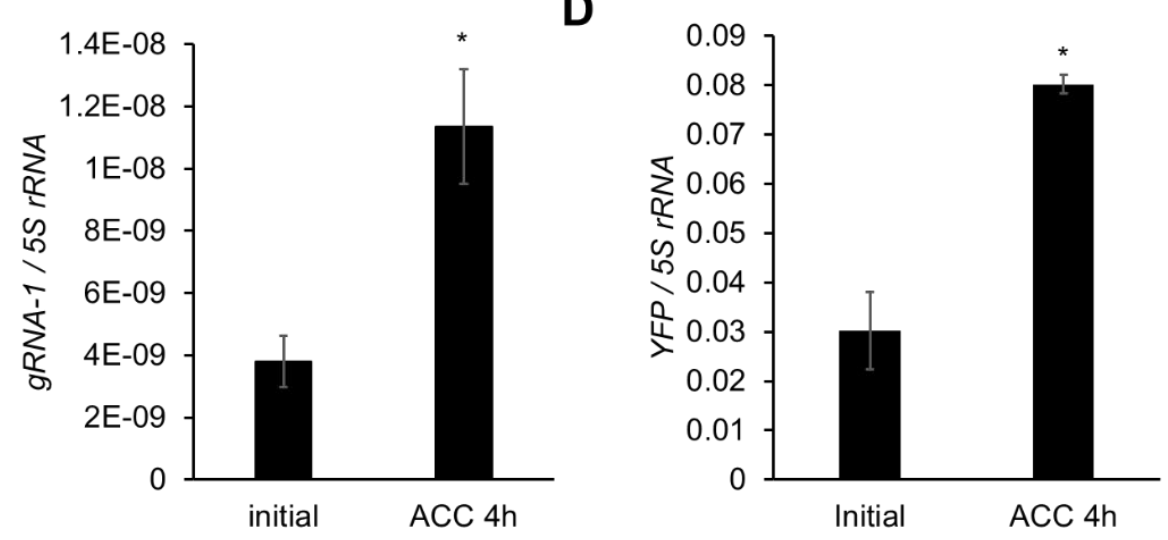

Figure 6. Characterization of an ethylene inducible orthogonal control system. A. OCS1-9 circuit ( $g R N A-1$ is expressed by ethylene inducible EBS promoter) B. Time course fluorescence microscope images showing Agrobacterium mediated transient expression of OCS1-9 in Nicotiana benthamiana leaves after induction with 10 $\mu \mathrm{M}$ ACC. Scale bars: $200 \mu \mathrm{m}$ C and D. qPCR quantification of $g R N A-1$ (C) and YFP(D) expression before and after induction with ACC, where both show similar levels of induction demonstrating that the relative change in $g R N A-1$ expression (ethylene induction) results in the differential activation from the pATF-1 promoter. Error bars: S.D. $(n=3$, independent replicates), Asterisks indicate statistical significance in a student t-test $(P<0.05)$. 
A

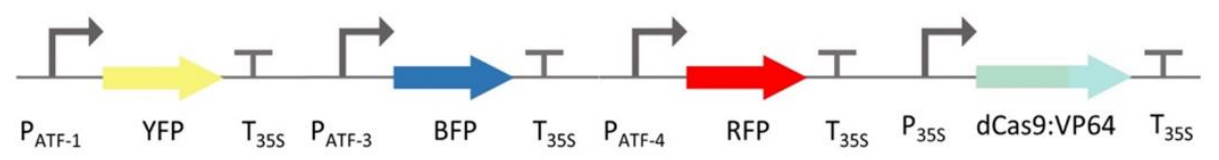

B
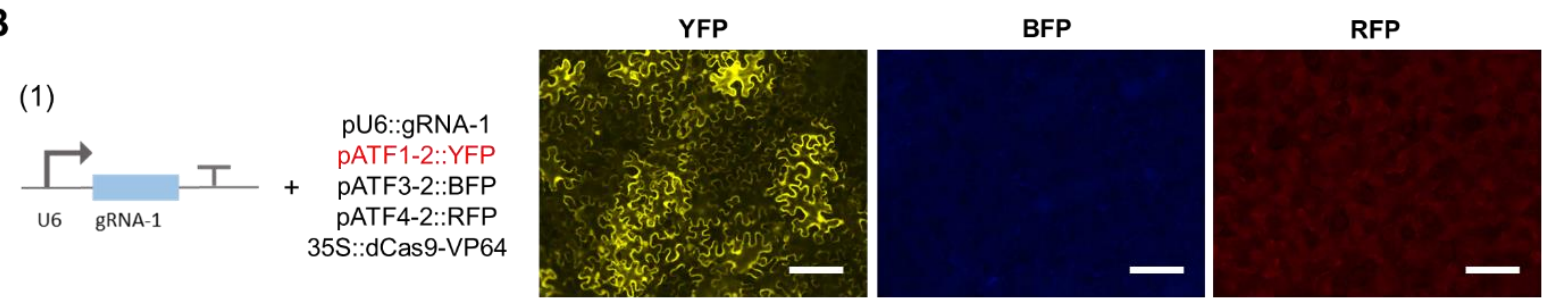

(2)

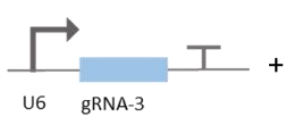

pU6::gRNA-3 pATF1-2::YFP PATF3-2::BFP pATF4-2::RFP 35S::dCas9-VP64
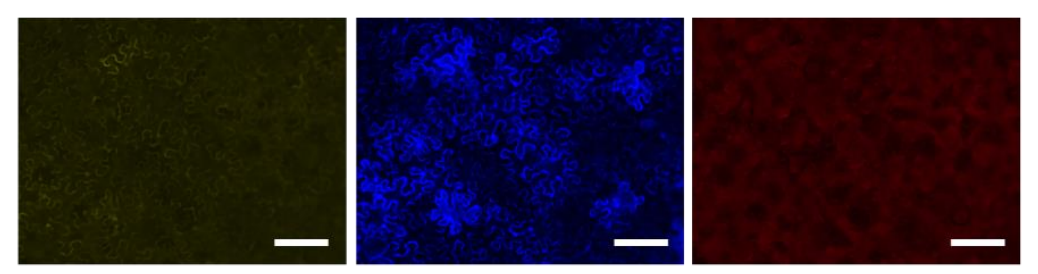

(3)
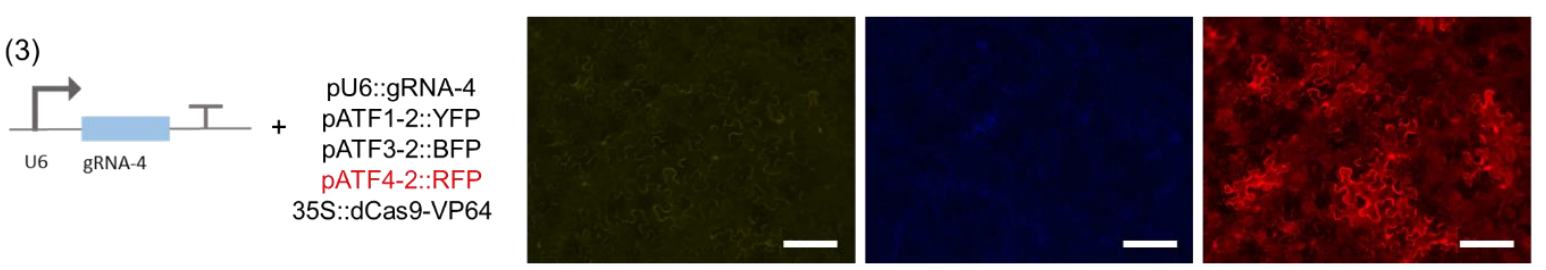

C
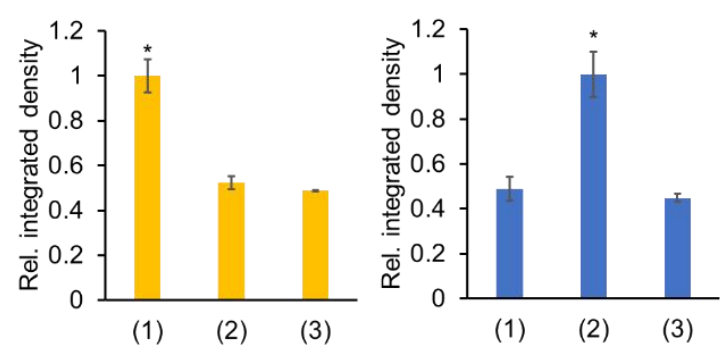

(1) (2) (3)

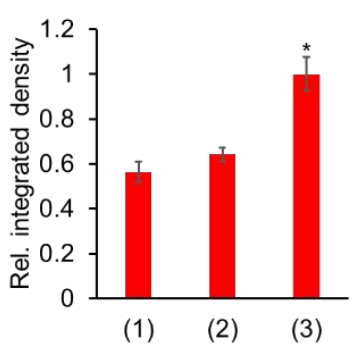

Figure 7. Degree of orthogonality of synthetic promoters. A. OCS circuit containing all three synthetic promoters (pATF-1, pATF-3 and pATF-4) driving three different reporter genes namely YFP, BFP and RFP respectively with a single gRNA expressed one at a time under the control of U6 promoter. B. Fluorescence microscope images showing Agrobacterium mediated transient expression of OCS constructs in Nicotiana benthamiana leaves. Scale bars: $200 \mu \mathrm{m}$ C. As observed from the fluorescence images, only the specific gRNA:pATF pair is active, thus demonstrating that the synthetic promoters are mutually orthogonal Relative integrated density of each fluorescence signal (shown in panel B). Integrated density was measured by image $\mathrm{J}$ software and normalized to the highest value. Error bars: S.D. ( $n=3$, independent replicates). Asterisks indicate statistical significance in a student t-test $(\mathrm{P}<0.05)$. 
A

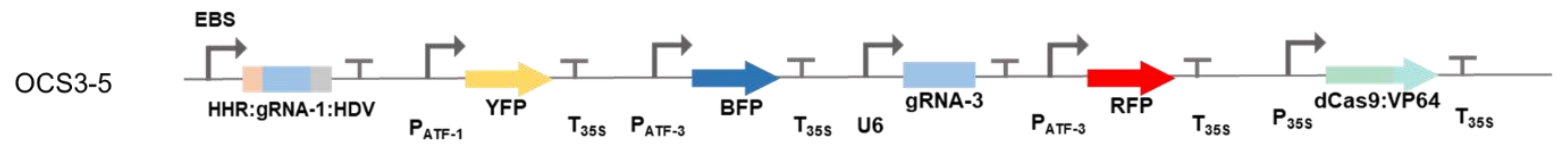

B
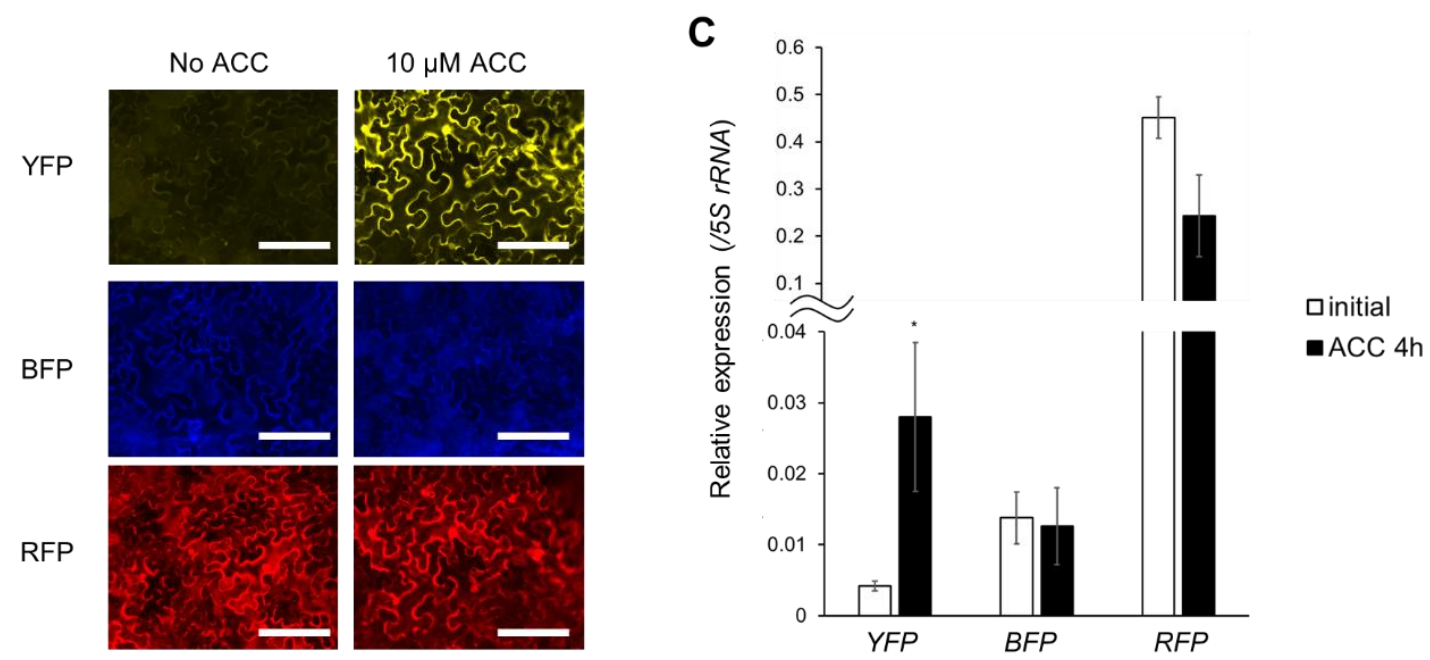

Figure 8. Design and characterization of a ratiometric circuit. A. OSC3-5 contains YFP which is inducible by ACC (pATF-1), while BFP and RFP are constitutively expressed under the control of pATF-3 via the constitutive expression of gRNA-3. B. Fluorescence microscope images showing Agrobacterium mediated transient expression of the ratiometric OCS construct (OCS3-5) in Nicotiana benthamiana leaves with or without $10 \mu \mathrm{M}$ ACC. Scale bars: $200 \mu \mathrm{m}$ C. qPCR quantification of $Y F P, B F P$ and $R F P$ shows that YFP is induced after the treatment with ACC while the expression of BFP and RFP remains unchanged before or after ACC induction. Error bars: S.D. $(n=4$, independent replicates). An asterisk indicates statistical significance in a student t-test ( $P$

$771<0.05)$. 